\title{
Demências: da investigação ao diagnóstico
}

\author{
Investigation and diagnostic evaluation of a patient with dementia
}

\author{
Jacy Bezerra Parmera ${ }^{1}$, Ricardo Nitrini ${ }^{2}$
}

Parmera JB. Nitrini R. Demências: da investigação ao diagnóstico / Investigation and diagnostic evaluation of a patient with dementia. Rev Med (São Paulo). 2015 jul.-set.;94(3):179-84.

\begin{abstract}
RESUMO: Atualmente, define-se demência como condição na qual ocorre decréscimo cognitivo comparado a um nível prévio do indivíduo, com comprometimento de suas funções sociais e funcionais. Na avaliação em um paciente com comprometimento cognitivo e com uma possível síndrome demencial, a anamnese clínica e o exame neurológico são de extrema importância, e os exames complementares. Com o avanço dos biomarcadores, novas técnicas de diagnóstico evoluíram proporcionando maior acurácia na avaliação, contudo a indicação destas ainda está restrita a casos específicos e no âmbito das pesquisas científicas.
\end{abstract}

Descritores: Demência/diagnóstico; Doença de Alzheimer; Demência vascular, Comprometimento cognitivo leve.

\begin{abstract}
Dementia is defined as an acquired decline of cognitive abilities sufficient to result in social or occupational impairment. The key goals of a clinical evaluation are to estabilish the impaired areas of cognition, the severity of impairment and functional consequences. Clinical diagnostic of dementia are evolving, particularly due to new methods such as functional neuroimaging and biomarkers, although the availability does not replace or diminish the need for a thorough evaluation.
\end{abstract}

Keywords: Dementia/diagnosis; Alzheimer disease; Dementia, vascular; Mild cognitive impairment.

\section{EPIDEMIOLOGIA DAS DEMÊNCIAS}

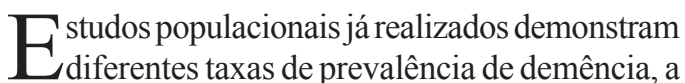
depender dos critérios utilizados e da população avaliada. Entretanto, sabe-se que os quadros demenciais são muito comuns na população idosa, com prevalência que dobra a cada cinco anos aproximadamente, a partir dos 65 anos $^{1}$. Há também revisões sistemáticas demonstrando que, apesar da heterogeneidade da prevalência de demência, em todos os estudos houve aumento com a idade. Segundo Ferri et al. ${ }^{2}$ utilizando a divisão proposta pela $\mathrm{WHO}$ em regiões pelo mundo, a prevalência de demência varia entre 1,6\% na África a 6,4\% na América do Norte, em indivíduos com idade igual ou superior a 60 anos.

Em todos os estudos da América Latina, por sua vez, há um aumento da prevalência com a idade e a maior causa de demência é a Doença de Alzheimer (DA). Na publicação de Nitrini et al. ${ }^{3}$ dados de oito estudos de base populacional realizados na América Latina foram analisados em conjunto. Pôde-se observar que demência foi mais prevalente entre os analfabetos $(15,7 \%)$, entre os

1. Neurologista pela FMUSP, Complementação especializada em Neurologia Cognitiva e Comportamental no Hospital das Clínicas da FMUSP; colaboradora do Grupo de Distúrbios do Movimento da FMUSP. E-mail: jacy_parmera@hotmail.com

2. Professor Titular de Neurologia da FMUSP; Coordenador do Centro de Referência de Distúrbios Cognitivos (CEREDIC) e do Grupo de Neurologia Cognitiva e do Comportamento (GNCC) do Hospital das Clínicas da FMUSP. E-mail: rnitrini@uol.com.br.

Endereço para correspondência: Av. Dr. Enéas de Carvalho Aguiar, 647. Cerqueira César, São Paulo, SP. CEP: 05403.900. 
mais idosos, porém, em relação aos estudos realizados em países desenvolvidos, houve uma maior prevalência nas faixas etárias mais jovens. A prevalência geral, contudo, foi semelhante aos países de maior renda $(7,13 \%)$.

Em relação à incidência, no Brasil temos dados de dois estudos, com resultados diferentes. Um deles realizado na cidade de Porto Alegre, com taxa de 14,8/1000/ano em idosos acima de 65 anos de $\mathrm{DA}^{4}$ e outro na cidade de Catanduva (interior de São Paulo), com incidência de 7,7/1000/ano para DA e 13,8/1000/ano para demência ${ }^{5}$. Neste último estudo, a incidência de demência dobrou a cada cinco anos, sem diferença por sexo, mas houve uma maior incidência de demência em mulheres em idade mais avançada. Também se observou uma tendência à maior incidência entre analfabetos.

\section{DEMÊNCIA: INTRODUÇÃO E CONCEITO}

Atualmente, define-se demência como condição na qual ocorre decréscimo cognitivo comparado a um nível prévio do indivíduo, com comprometimento de suas funções sociais e funcionais. Há várias propostas para a definição a ser adotada de demência, entre elas a do Diagnostic and Statistical Manual of Mental Disorders (DSM IV) ${ }^{6}$, na qual o comprometimento de memória é exigido necessariamente na apresentação, ademais deverá haver declínio em relação a um padrão prévio cognitivo e não poderá haver delirium concomitante durante a avaliação. Outras, como a do Grupo de Trabalho do National Institute on Aging (NIA) e Alzheimer's Association (AA) ${ }^{7}$, propõem novos critérios que não exigem comprometimento de memória inicialmente, possibilitando sua aplicação para várias etiologias de demência. Esses últimos são, portanto, os critérios recomendados para a aplicação no Brasil pela Academia Brasileira de Neurologia ${ }^{8}$.

$\mathrm{Na}$ avaliação inicial dos pacientes, idealmente, seria interessante comparar longitudinalmente suas performances cognitivas para se realizar com acurácia tal diagnóstico, contudo, como esses dados são de difícil obtenção no âmbito prático, determina-se o diagnóstico através de exames cognitivos com escore e resultados padronizados para a média da população.

Considerando a crescente prevalência populacional, as demências tornam-se uma questão primordial em saúde pública, ocasionando gastos diretos e indiretos, com aposentadorias, internações, assim como repercussões em trabalhadores ativos por acometimento de familiares que requerem seus cuidados. Medidas públicas de saúde e conscientização tornam-se urgentes para o cuidado e atendimento dessa parcela da sociedade em ascensão demográfica comparativa aos outros grupos etários.

Podem-se dividir as demências em categorias diversas para estudos e pesquisas direcionadas, a exemplo das divisões em degenerativas e não degenerativas, corticais e subcorticais, com início precoce (ou pré-senil - antes dos 65 anos de idade) e tardio (a partir dos 65 anos), reversíveis ou irreversíveis, e rapidamente ou lentamente progressivas. Em sua maior parte, as demências têm início insidioso e evolução lenta, entretanto as mais diversas formas de apresentação se fazem possíveis e uma acurada suspeição diagnóstica em relação às diversas formas de apresentação das síndromes demenciais é imprescindível para evitar erros ou atraso no diagnóstico de doenças potencialmente tratáveis.

\section{INVESTIGAÇÃO INICIAL}

Para iniciar uma avaliação em um paciente com comprometimento cognitivo e com uma possível síndrome demencial, a anamnese clínica e o exame neurológico são de extrema importância. Não raramente, nesses casos, o transtorno cognitivo, o qual pode se apresentar como distúrbios de linguagem, memória, ou mesmo como anosognosia (incapacidade de reconhecer a própria limitação) o impede de relatar com precisão suas dificuldades, e por isso o relato de um acompanhante, de preferência alguém de estreito convívio com o paciente em questão, é de suma importância.

As metas chave da evolução clínica primária devem ser estabelecer se uma síndrome demencial está presente ou não, caracterizar os domínios cognitivos acometidos, a gravidade desse acometimento assim como seu prejuízo funcional, e por fim determinar sua etiologia para definir o tratamento adequado. Por vezes mais de uma avaliação ou consulta é necessária para abarcar todos esses aspectos, e várias entrevistas com diferentes familiares são necessárias para caracterizar adequadamente a patologia em investigação.

O processo de anamnese necessita abordar inicialmente o paciente e após isso entrevistar o informante, sempre que possível na ausência daquele, sobretudo quando se encontrar em fases leves ou moderadas da doença, podendo haver certa crítica conservada em relação à própria condição, e assim tal exposição poderia gerar estresses e conflitos desagradáveis. Além disso, algumas alterações comportamentais como hipersexualidade ou desinibição podem acarretar constrangimento e inibir o informante de relatar adequadamente o panorama do quadro investigado.

Durante a entrevista é interessante já observar algumas possíveis alterações de linguagem, como a fluência ao falar, se ocorrem pausas ou dificuldade de nomeação, assim como aspectos da memória como dificuldade de lembrar onde guardou objetos, repetir as mesmas histórias várias vezes para pessoas de seu convívio ou mesmo expressar desculpas por não se lembrar de determinados fatos "por não serem importantes", algo observado em pacientes com doença de Alzheimer (DA). Observar 
também a presença de confabulações e se há percepção das próprias dificuldades. Por outro lado, quando as queixas de memória são muito bem pontuadas e isso parece trazer bastante medo em relação a certo diagnóstico, um quadro comportamental como ansiedade ou depressão subjacente é sugerido.

Faz-se de extrema relevância questionar sobre o modo de evolução do declínio cognitivo, dessa forma, se o início for abrupto aventam-se algumas etiologias, sobretudo a vascular como causa da demência, que evolui às vezes de característica forma em degraus, mas também encefalites ou traumas podem ter início abrupto. Se o acometimento for progressivo, a etiologia degenerativa é a mais provável, com curso em geral insidioso, embora haja eventualmente a falsa percepção por parte de familiares que o déficit iniciou-se de forma súbita, quando eles atribuem a algum evento pontual, como uma internação ou cirurgia, ou evento pessoal traumático para o paciente, como falecimento de familiar. Sendo o quadro progressivo, é essencial definir o tempo médio de evolução, a fim de se distinguirem as demências rapidamente progressivas - as quais se instalam em semanas a meses - como as doenças priônicas, das demências de instalação crônica, como a DA, a demência Frontotemporal (DFT) ou demência com Corpos de Lewy, entre outras.

Alterações comportamentais são comuns em pacientes com síndromes demenciais e devem ser bem explorados, pois podem fornecer pistas importantes para o diagnóstico, a exemplo da DFT variante comportamental, cujos sintomas iniciais são justamente mudanças de personalidade, como apatia, desinibição, impulsividade, perda de empatia, antes mesmo de ocorrerem outros sintomas cognitivos evidentes. Alucinações, principalmente visuais, por sua vez, sugeririam um quadro de demência com Corpos de Lewy, se já presentes desde a instalação do declínio neurológico. Vale salientar que tanto apatia, como mudanças comportamentais e mesmo alucinações e delírios podem acontecer em vários tipos de demências, como a DA, entretanto o momento e a fase da doença em que ocorrem devem ser bem caracterizados para ajudar a determinar o diagnóstico. Distúrbios de sono, adicionalmente, aliados aos sintomas comportamentais, são bastante comuns, como o distúrbio comportamental do sono REM na demência da Doença de Parkinson e na demência com corpos de Lewy, ou a Apneia Obstrutiva do Sono, que pode piorar ou até gerar distúrbios cognitivos existentes, e requerem avaliação assim como tratamento conjunto com a doença de base.

Ainda na anamnese, é preciso investigar adequadamente os antecedentes pessoais, tais como doenças sistêmicas (doenças tireoidianas, hepáticas, reumatológicas, cardiovasculares), doenças neurológicas prévias, medicações utilizadas (a exemplo de antipsicóticos ou anticolinérgicos) ou hábitos de vida como etilismo. Questionar sobre história familiar de demência assim como história de consanguinidade, sobretudo para um paciente que possua quadro comportamental ou cognitivo de início prévio aos 65 anos.

No intuito de caracterizar adequadamente o declínio funcional, é adequado avaliar as atividades de vida diária, as básicas (higiene, alimentar-se sozinho, vestir-se sozinho, continência urinária e fecal) e as instrumentais (preparar refeições, pagar contas, cuidar das próprias finanças, limpar a casa, fazer compras), sempre comparando com o nível cognitivo prévio do indivíduo avaliado.

Após uma anamnese satisfatória, o próximo passo constitui o exame neurológico geral e cognitivo aprofundados. Durante o exame neurológico completo, devem-se buscar sinais importantes como parkinsonismo, outros transtornos do movimento, alterações na motricidade ocular, alterações de sensibilidade profunda, presença de reflexos primitivos, pesquisa de disautonomia, alterações da marcha, entre outros que possam sugerir uma determinada etiologia. O exame neurológico cognitivo, por sua vez, deve abranger todos os domínios - linguagem, funções executivas, memória, funções visuo-espaciais, praxias, gnosias - aliados a testes de rastreio como o miniexame do estado mental, bateria breve de rastreio cognitivo, bateria do CERAD, entre outros.

Embora não seja obrigatória para o diagnóstico nosológico da demência, Avaliação Neuropsicológica abrangente pode ajudar em determinadas situações, como em apresentações pouco usuais, assim como para avaliar quais seriam os domínios cognitivos mais afetados e sua progressão, além de poder detectar declínio em casos mais sutis ou em pacientes de alta escolaridade cujos testes de rastreio não foram suficientes para o diagnóstico.

Durante a investigação complementar, após anamnese e exame físico, alguns exames iniciais são preconizados. O consenso da Academia Brasileira de Neurologia sugere uma ampla investigação para se afastarem causas secundárias e tratáveis, além de realizar exames de imagem como tomografia de crânio ou, preferencialmente, se possível, ressonância magnética de encéfalo ${ }^{9}$. As causas reversíveis e tratáveis principais seriam: deficiência de vitamina B12 e folato, hipotireoidismo, depressão (pseudodemência), doenças infecciosas (sífilis e tuberculose), hidrocefalia de pressão normal, tumores, hematoma subdural, intoxicação medicamentosa, etilismo, vasculites, hiperparatireoidismo, insuficiência adrenal, renal, hepática e pulmonar. Consideram-se como obrigatórios os exames laboratoriais: hemograma, creatinina, hormônio tireoestimulante, albumina, transaminases hepáticas, vitamina B12, reações sorológicas para sífilis, e, para pacientes com menos de 60 anos e quadro sugestivo, testagem para o vírus HIV. Deve-se ter em mente que comorbidades são muito comuns em idosos, e que a presença de condições como anemia, hipovitaminose, hipotireoidismo ou medicamentos em uso podem agravar a síndrome demencial causada pela DA ou 
por outra doença.

Da mesma forma, a Academia Americana de Neurologia recomenda testagem de vitamina B12, hormônios tireoidianos, creatinina e hemograma. Testes como sorologia para HIV, assim como coleta do líquido cefalorraquidiano, por sua vez, ficam reservados para situações especiais e não para todos os casos. Alguma imagem estrutural como tomografia de crânio ou ressonância de crânio é igualmente recomendada. Adicionalmente, eletroencefalograma não é recomendado de rotina, apenas para casos específicos ${ }^{10}$.

\section{DEMÊNCIAS: DIAGNÓSTICOS DIVERSOS}

\section{Doença de Alzheimer}

A DA é a forma mais frequente de demência em idosos e sua prevalência aumenta de forma expressiva com a idade. O envelhecimento é o fator de risco mais importante para o desenvolvimento da DA, embora outros fatores como histórico familiar e genética também influenciem. Sabe-se, entretanto, que as formas familiares de padrão autossômico dominante, nas quais geralmente o início é pré-senil (antes dos 65 anos de idade), perfazem menos de $5 \%$ do total de casos. Outro aspecto genético considerável é a presença do alelo E4 do gene da apolipoproteína E, a qual é de alta frequência nos casos de DA, tanto esporádico como familiar.

Com relação aos aspectos patológicos, na DA há atrofia cortical macroscópica, sobretudo em áreas do córtex associativo neocortical e regiões temporais mesiais (hipocampais). Na microscopia, observam-se emaranhados neurofibrilares intracelulares com proteína TAU e deposição de peptídeo B-amilóide em placas neuríticas extracelulares. Vale salientar que o exame neuropatológico constitui o padrão-ouro para o diagnóstico de DA, observando-se a distribuição e a quantidade das lesões descritas.

Inicialmente, a principal característica do quadro clínico é o comprometimento de memória episódica, como, por exemplo, dificuldade de lembra-se de fatos recentes, repetição de perguntas e perda de objetos pessoais. Às vezes, apenas começa como comprometimento cognitivo leve amnéstico, quando ainda não há comprometimento funcional e, portanto, síndrome demencial. Ocorre, posteriormente, envolvimento de área corticais associativas frontais, temporais e parietais, surgindo sintomas outros comportamentais e cognitivos. Existem outras formas clínicas de início da DA, inclusive formas não-amnésticas, sobretudo em casos de DA pré-senil.

Além das dificuldades para realizar atividades funcionais da vida diária, os sintomas comportamentais são bastante prevalentes, sobretudo apatia, depressão e agitação psicomotora. Com o avançar da doença há prejuízo para realizar atividades básicas da vida diária, o paciente torna-se incapaz de realizar sua própria higiene pessoal, de alimentar-se ou vestir-se. É possível durante a evolução do quadro clínico encontrar-se parkinsonismo, mioclonias, e até mesmo crises epilépticas.

O diagnóstico ainda é basicamente clínico, embora haja marcante avanço de marcadores biológicos para as fases iniciais da patologia. Baseia-se, sobretudo, nas alterações cognitivas, comportamentais e funcionais aferidas por baterias cognitivas e exame neurológico, associadas à exclusão de outras causas de demência, através exames complementares, laboratoriais e de imagem. Os marcadores biológicos de maior acurácia atualmente são a neuroimagem funcional e estrutural ressonância magnética de encéfalo tipicamente demonstra atrofia de estruturas temporais mesiais e dilatação do corno temporal dos ventrículos laterais - e a dosagem de proteínas no LCR, em que há aumento da proteína TAU e TAU hiperfosforilada, com diminuição de B-amilóide. $\mathrm{Na}$ neuroimagem funcional, a tomografia por emissão de pósitrons (PET) com fluorodesoxiglicose revela tipicamente o hipometabolismo temporoparietal posterior. Um método ainda mais avançado, que será de grande utilidade em alguns anos, é o PET com radiotraçador que utiliza Carbono-11(PIB - Pittsburgh compound B) ou Fluor-18 (Florbetapir, Flutemetamol) que detecta depósitos de amiloide in vivo.

\section{Demência vascular}

É reconhecido hoje o fato de a doença vascular tratar-se do fator de risco mais identificável para demência, ao lado da idade, e de ser passível de controle.. Atualmente, o critério para demência vascular mais aceito é o do National Institute of Neurological Disorders and Stroke - Association Internationale pour la reserche et L'enseignement en Neurosciences (NINDS-AIREN), no qual constam que deve haver demência, associada a doença cerebrovascular - esta, por sua vez, definida pela presença de alterações ao exame neurológico ou através de exames de imagem - e uma relação estabelecida entre ambos. Esta relação pode ser, por exemplo, por início de uma síndrome demencial dentro de três meses após um AVC reconhecido, ou por uma deterioração abrupta das funções cognitivas, ou por déficits cognitivos com aparecimento em "degraus". Algumas características reforçam o diagnóstico, como presença precoce de distúrbios da marcha, tais como marcha em petit-pas ou apráxica, relato de desequilíbrio, paralisia pseudobulbar e urgência urinária precoce, além de mudanças na personalidade ou humor. A presença de déficit amnéstico precoce e progressivo, assim como ausência de lesões vasculares em exames de imagem ou ausência de sinais neurológicos focais tornam o diagnóstico menos provável.

A fisiopatologia no comprometimento cognitivo 
vascular (CCV), termo bastante utilizado atualmente, envolve alterações em vasos cerebrais pequenos, acometendo a substância branca cerebral, chamada de leucoaraiose, assim como infartos pequenos estratégicos ou extensos, e lesões subcorticais extensas, com comprometimento de redes neurais.

Os sintomas são bastante variáveis, dependendo do território vascular afetado, e a velocidade de progressão também varia entre os acometidos. Nos infartos subcorticais predominam alterações de funções executivas, podendo também estar presentes alterações de memória, linguagem, praxias, entre outros, como alucinações, delírios, alterações da marcha e esfincterianas.

Os principais fatores de risco para doença vascular cerebral são hipertensão arterial, diabetes mellitus, dislipidemia e tabagismo. Estudos epidemiológicos têm demonstrado que estes também são fatores de risco para DA, e estudos neuropatológicos mais atuais demonstram que a associação de alterações vasculares cerebrais e doença de Alzheimer é muito frequente. Ainda é incerto, entretanto, se os fatores de risco para doença vascular aumentam o risco de DA ou agravam e antecipam as manifestações clínicas da DA devido à presença concomitante de lesões vasculares.

A taxa de progressão também é variável, e pode progredir mais devagar do que a DA. A taxa de mortalidade, entretanto, é mais alta, embora com o advento de melhores terapêuticas para o AVC e para os fatores de risco tal constatação venha a ser mudada nos próximos anos.

\section{Demência frontotemporal}

A Degeneração Lobar FrontoTemporal (DLFT) corresponde a um grupo heterogêneo de diagnósticos neuropatológicos e também de diferentes síndromes clínicas. Trata-se da segunda causa de demência degenerativa mais frequente na população com menos de 65 anos (a primeira é a DA). Quando a alteração comportamental é predominante, denomina-se variante comportamental da DLFT. Se, por sua vez, ocorrer principalmente alteração das habilidades linguísticas, têm-se as afasias progressivas primárias (demência semântica e afasia progressiva nãofluente).

$\mathrm{Na}$ DLFT há macroscopicamente atrofia assimétrica dos lobos frontais e temporais, e, atualmente, são reconhecidos três tipos de proteínas anômalas DLFT-TAU, DLFT- TDP (proteína TDP-43) e DLFT-FUS (fused in sarcoma). Com relação aos aspectos genéticos, aproximadamente $40 \%$ dos pacientes possuem história familiar positiva, e cerca de 10 a $20 \%$ apresentam padrão de herança autossômica dominante.

$\mathrm{Na}$ variante comportamental há sintomas decorrentes de lesão em áreas de lobo pré-frontal, como redução de empatia, afeto inapropriado, irritabilidade e perda de autocrítica. Apatia é marcadamente presente no quadro clínico, associada à lesão na parte anterior do giro de cíngulo; alterações da preferência alimentar, hipersexualidade e comportamento de utilização estão ligadas por sua vez a acometimento orbitofrontal. Pacientes com o lobo temporal direito acometido apresentam comportamento antissocial, hiperreligiosidade e comportamento compulsivo. No exame cognitivo é detectada disfunção executiva, com relativa preservação da memória e habilidades visuoespaciais. Sinais e sintomas de parkinsonismo estão associados a cerca de $20 \%$ dos casos. A demência semântica é uma síndrome clínica com distúrbio da linguagem fluente, ocorre perda progressiva do conhecimento de palavras e compreensão delas, com parafasias semânticas. Patologicamente, há atrofia temporal anterior assimétrica e a maior parte é composta por TDP43.

A afasia progressiva não-fluente é caracterizada por agramatismo, apraxia de fala e parafasias fonêmicas. Percebe-se redução da produção de palavras e dificuldade de articulação.

Para o diagnóstico é importante a pesquisa com exames de imagem - ressonância de encéfalo e PET-FDG- e em casos pré-senis, o exame do líquido cefalorraquidiano também é indicado.

\section{Demência com corpos de Lewy}

Hoje é considerada a segunda causa de demência degenerativa, atrás apenas da $\mathrm{DA}^{11}$. As características principais são um quadro demencial progressivo com parkinsonismo em geral simétrico e com pouco tremor, alucinações visuais recorrentes e flutuação cognitiva. A resposta do parkinsonismo à levodopa é pequena.

Nos critérios diagnósticos ${ }^{12}$ existe uma regra arbitrária na qual a demência deve anteceder ou suceder no máximo em um ano o sintomas parkinsonianos, para distinguir da demência na Doença de Parkinson. A flutuação cognitiva é o sintoma mais prevalente na DCL, podendo ocorrer em até $90 \%$ dos pacientes. Desta forma, há sonolência variável ao longo do dia e diferentes respostas cognitivas da atenção e do nível de consciência.

As alucinações visuais já podem surgir desde as fases iniciais da doença, o que ajuda a diferir da DA, na qual aparecem de forma mais tardia.

Existem características que apoiam o diagnóstico, entre elas estão a hipersensibilidade aos neurolépticos, quedas repetidas, distúrbio comportamental do sono REM, uma parassonia na qual ocorre atuação durante os sonhos por ausência da perda de tônus desse período. Este distúrbio do sono está presente em outras doenças e atualmente é atribuído às alfa-sinucleinopatias, não apenas à DCL.

Para o diagnóstico, além da triagem inicial, o exame cognitivo demonstra pior desempenho em 
testes de atenção, habilidades visuoespaciais e praxias construcionais, em relação à memória relativamente preservada. A neuroimagem funcional pode ajudar no diagnóstico. O SPECT e o PET cerebral demonstram alteração de perfusão ou do metabolismo em regiões parietais posteriores e occipitais.

\section{CONCLUSÃO}

Para definir uma síndrome demencial é necessário declínio cognitivo suficiente para gerar prejuízo funcional em relação a um nível prévio do indivíduo. Hoje são

\section{REFERÊNCIAS}

1. Jorm AF, Korten AE, Henderson AS. The prevalence of dementia: a quantitative integration of the literature. Acta Psychiatr Scand. 1987;76(5):465-79. doi: 10.1111/j.16000447.1987.tb02906.x.

2. Ferri CP, Prince M, Brayne C, Fratiglioni L, Ganguli M, Hall K, Hasegawa K, Hendrie H, Huang Y, Jorm A, Mathers C, Menezes PR, Rimmer E, Scazufca M, and Alzheimer's Disease International. Global prevalence of dementia: a Delphi consensus study. Lancet. 2005;366(9503):2112-7. doi: 10.1016/S0140-6736(05)67889-0.

3. Nitrini R, Bottino CM, Albala C, Custodio Capuñay NS, Ketzoian C, Llibre Rodriguez JJ, Maestre GE, RamosCerqueira AT, Caramelli P. Prevalence of dementia in Latin America: a collaborative study of population-based cohorts. Int Psychogeriatr. 2009;21(4):622-30. doi: 10.1017/ S1041610209009430.

4. Volpe FM, Abrantes MM, Capanema FD, Chaves JG. The impact of changing health indicators on infant mortality rates in Brazil, 2000 and 2005. Rev Panam Salud Publica. 2009;26(6):478-84. http://dx.doi.org/10.1590/S102049892009001200002.

5. Nitrini R, Caramelli P, Herrera E Jr, Bahia VS, Caixeta LF, Radanovic M, Anghinah R, Charchat-Fichman H, Porto CS, Carthery MT, Hartmann AP, Huang N, Smid J, Lima EP, Takada LT, Takahashi DY. Incidence of dementia in a community-dwelling Brazilian population. Alzheimer Dis Assoc Disord. 2004;18(4):241-6. Available from: http:// goo.gl/Sggn8O.

6. American Psychiatric Association. Diagnostic and statistical manual of mental disorders. 4th ed. Washington, DC: APA; 1994.

7. Frota NAF, Nitrini R, Damasceno BP, Forlenza O, DiasTosta E, Silva AB, Herrera Junior E, Magaldi RM. Criteria for the diagnosis of Alzheimer's disease: recommendations of the Scientific Department of Cognitive Neurology and exigidos acometimento de dois domínios cognitivos afetados, não necessariamente a memória na maior parte dos critérios em voga.

Faz-se necessária investigação extensa inicial, sobretudo em casos pré-senis (antes dos 65 anos), casos de demências potencialmente reversíveis e demências rapidamente progressivas.

Com o atual avanço da neuroimagem funcional e dos biomarcadores, novos critérios para as diferentes demências tenderão a adotar esse arsenal terapêutico no âmbito clínico e não apenas como critérios de pesquisa.

Aging of the Brazilian Academy of Neurology. Dement Neuropsychol. 2011;5(3):146-52. Available from: http:// www.demneuropsy.com.br/detalhe_artigo.asp?id=289.

8. Nitrini R, Caramelli P, Bottino CMC, Damasceno BP, Brucki SMD, Anghinah R. Diagnóstico de Doença de Alzheimer no Brasil. Critérios diagnósticos e exames complementares. Arq Neuropsiquiatr. 2005;63(3):713-9. http://dx.doi.org/10.1590/S0004-282X2005000400033.

9. Nitrini R1, Caramelli P, Bottino CM, Damasceno BP, Brucki SM, Anghinah R; Academia Brasileira de Neurologia. Diagnosis of Alzheimer's disease in Brazil: cognitive and functional evaluation. Recommendations of the Scientific Department of Cognitive Neurology and Aging of the Brazilian Academy of Neurology. Arq Neuropsiquiatr. 2005;63(3A):720-7. http://dx.doi.org/10.1590/S0004282X2005000400034.

10. Knopman DS, DeKosky ST, Cummings JL, Chui H, Corey-Bloom J, Relkin N, Small GW, Miller B, Stevens JC. Practice parameter: diagnosis of dementia (an evidence-based review).Report of the Quality Standards Subcommittee of the American Academy of Neurology. Neurology. 2001;56(9):1143-53. doi: http:/dx.doi. org/10.1212/WNL.56.9.1143.

11. Hansen LA, Masliah E, Galasko D, Terry RD. Plaque-only Alzheimer disease is usually the Lewy body variant, and vice-versa. Int J Geriatr Psychiatry. 2003;18(11):988-93.

12. McKeith IG, Galasko D, Kosaka K, Perry EK, Dickson DW, Hansen LA, Salmon DP, Lowe J, Mirra SS, Byrne EJ, Lennox G, Quinn NP, Edwardson JA, Ince PG, Bergeron C, Burns A, Miller BL, Lovestone S, Collerton D, Jansen EN, Ballard C, de Vos RA, Wilcock GK, Jellinger KA, Perry $\mathrm{RH}$. Consensus guidelines for the clinical and pathologic diagnosis of dementia with Lewy bodies (DLB): report of the consortium on DLB international workshop. Neurology. 1996;47(5):1113-24. 